\title{
A remote lab to support e-leaning on Programmable System-on-Chip (PSoC)
}

\author{
Manuel Felgueiras, André Fidalgo, Gustavo Alves \\ CIETI-LABORIS \\ Polytechnic Institute of Porto - School of Engineering \\ ISEP/IPP \\ Porto, Portugal \\ $\{$ mcf, anf, gca $\} @$ isep.ipp.pt
}

\author{
Gabriel Motta, Luis Schlichting, Golberi Ferreira \\ Instituto Federal de Santa Catarina \\ IFSC \\ Santa Catarina, Brazil \\ gabriel.v.motta@gmail.com \\ \{schlicht, golberi\}@ifsc.edu.br
}

\begin{abstract}
The design flow in analog and digital electronics are quite opposite as result of each area maturation state. So, are also the teaching methodologies, being challenging for both teachers and students. The electronic design in the digital field is centered in the use of microprocessor and FPGA based circuits using high level programing/configuring languages. In a different way, the analog design is traditionally based on the use of elementary components associated with macroblocks, such operational amplifiers in order to built-up the wanted mission circuit. Some analog programmable components, as the PSoC, are analogically configurable in a manner similar to those already used in the digital domain. The use of this type of components is not straightforward, being necessary to get some concepts traditionally not taught in the analog electronic classes. The training using PSoC is then indispensable to verify if the programmed circuit corresponds to the intended one.
\end{abstract}

The current work presents an innovative remote lab to support teaching of the PSoC.

Index Terms - Remote labs, PSoC, analog electronic design.

\section{INTRODUCTION}

The function of the engineering profession is to manipulate materials, energy and information [1]. Teaching the several subjects includes information about theory that must be complemented by the experimentation in order to build the knowledge. This completion can be a demonstration for something learned or for verifying if some experiment meets the specifications. Laboratories are essential to demonstrate laws or concepts related to a given subject [2]. In the past, traditional laboratories classes were based in lab rooms oriented by a teacher. In the last years the Remote Labs rising in importance by several reasons and are now seen as one unavoidable alternative in practical teaching [3].

The digital and analog electronic teaching includes the Electronic Design flow strategies. However these two areas are in very different state of development and so is the flow design methodology. In fact, the first is based in the use of programs/configuration using a standard language, in a hardware changeless approach. On the contrary, the second one is traditionally based in the use of typical circuit topologies adapted to each situation in where each change is made by hardware modification.

A few components such the Field Programmable Analog Arrays (FPAA) [4,5], or the Programmable System-on-Chip (PSoC) [6] allow to make the analog electronic design in a manner similar to the currently used in digital circuits, through a program/configuration. However the use of these components requires the knowledge of some specific concepts, unlike their digital counterpart, making its use a not straightforward operation. As such, practice on PSoCs circuits becomes very important to attain significant knowledge level in this field. This work presents a remote lab to support the eLearning on PSoC.

\section{ELECTRONIC TEACHING}

Teaching is a structured activity that encompasses several issues such as the reuse of earlier concepts to transmit new ones. One major limitation is that the several subjects to teach remain in a significant different state of development of its components and for so imposing different approach methodologies. This is the case of electronics subjects where the digital electronics present a very high development state when compared with analog electronics.

The first is based in the use of flexible circuits based on microprocessors (e.g. microcontrollers) or Field Programmable Gate Array (FPGA). The definition of the Mission Circuit is made by using normalized programing languages (e.g. C language) for microprocessor based circuits or Hardware Description Languages (HDL) (e.g. Verilog, VHDL) for FPGA. This methodology corresponds to the use of high level commands instructions which are associated to produce a program. This methodology is very appealing to the new generation of students and completely changeless at hardware level. In this way, the user can take advantage from the regular improvements in components features such new powerful instructions, speed, size etc. The second is traditionally based in the use of elementary components (e.g. resistors, capacitors, diodes, transistors, etc.) or macroblocks (e.g. Operational Amplifiers) that are associated to procedure the Mission Circuit. This methodology starts continually from the elementary and corresponds to constant reinvention is the wheel. These approaches are depicted in the figure 1 .

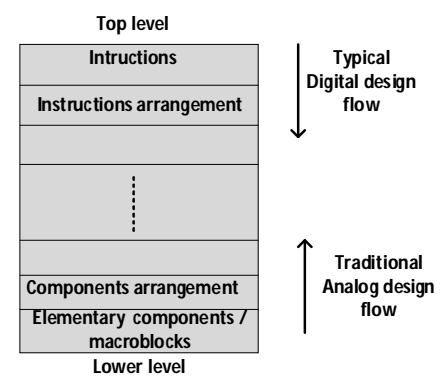

Figure 1. Design flow for analog and digital circuits. 
As seen, the digital area uses a top-down electronic design methodology whereas in the analog area uses a bottom-up one. Being opposite, this often bring some confusion to the student that prefer to remain only in the digital area, which is more high level and thus more close to the human way to think.

The PSoC is a programmable analog component that can be configured using the very same methodology used on digital flexible circuits. The figure 2 presents the PSoC development kit.

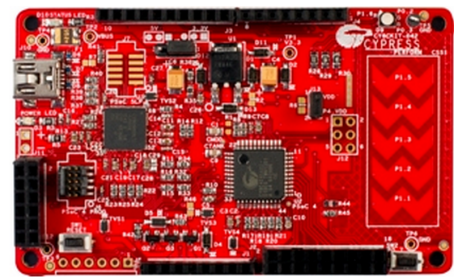

Figure 2. The PSoC development kit.

However, the use of this type of components is not straightforward, presenting some drawbacks that should be taken in account in the possible transition between the traditional manner of teaching analog design to the one using FPAA. It is necessary to establish some steps to make the use of the FPAA acceptable by the traditional student that comprehends the development of:

- A set of resources to clarify concepts

- A remote lab to practice with FPAA

- A set of resources to inform the student about his knowledge level

The first includes the development of writing notes, slides, flash movies, etc., to explain some notions related to analog design such as grounding (e.g. differences between ground and earth, power supply ground, analog ground, digital ground etc.), types of signals (digital, analog, quasi-digital), signals references (single-ended signal, double-ended signal), and switched-capacitor technology. All these concepts are important because they are intrinsically involved with the PSoC. For instance, it works with single supply and the analog ground is referred to a different voltage from the main power supply (e.g. analog ground $=1.5 \mathrm{~V}$ ). This part of the work is practically concluded and will be soon available to the community.

The second one is important to practice with PSoC without the need to buy a development kit. This point will be clarified in a subsequence section.

The third one is related to the resources that will be developed to help the student verify if his skill level matches the intended and will be based in the use of the MOODLE.

\section{THE PSOC REMOTE LAB}

The developed PSoC remote lab intends to provide a simple platform to remotely programing the PSoC and verify if the actual Mission Circuit matches the intended one. This platform includes several blocks as depicted in figure 3 .

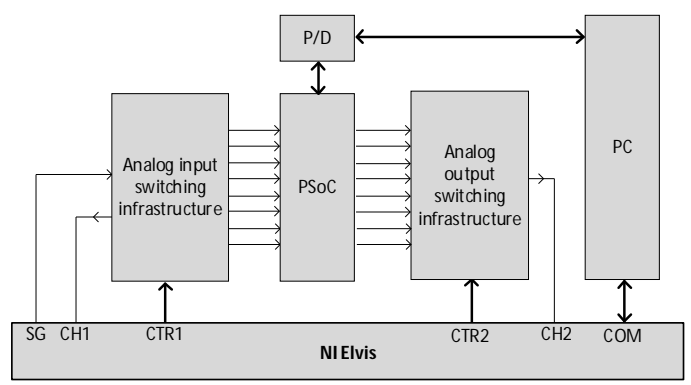

Figure 3. The PSoC remote lab infrastructure.

The platform core is the PSoC. Its input lines are connected to the analog input switching infrastructure whereas the output lines are connected to analog output switching infrastructure. The NI ELVIS station includes a Signal Generator (SD), two channels oscilloscope (CH1, $\mathrm{CH} 2$ ) and a set of digital output signals to control each analog switching infrastructure. The input switching infrastructure one is responsible for selecting the signal from the SD and the $\mathrm{CH} 1$ to the wanted PSoC input. The output switching infrastructure selects which output will be monitored by $\mathrm{CH} 2$. A personal Computer (PC) must have applets to both manage the NI ELVIS and communicate with the Program/Debug (P/D) circuit specific oriented circuit.

The presented platform allows developing a significant number of experimental tests in the PSoC.

\section{CONCLUSION}

Teaching analog electronic design subjects involves a methodology other than the used in the digital electronic design, mainly due to the different state of development of the electronic components from each area. In the digital is currently used the top-down approach whereas the bottom$u p$ is used in the analog area. The first is easier to use, not only because it is closer to the human brain but also its hardware is changeless. This work presents a remote lab to support learning in PSoC. After having attained some basics of PSoC, the user will be able to verify if the developed configuration matches the intended one.

\section{REFERENCES}

[1] Feisel, L., Rosa, A., "The role of the Laboratory in the Undergraduate Engineering Education", Journal of Engineering Education, January, 2005.

[2] Silva, R. ; Moreira, R. ; Gouveia, L. ; Felgueiras, M., "Unblinding a Braille machine - A case study of reverse problem-based learning". Proceedings of the 2013 1st International Conference of the Portuguese Society for Engineering Education (CISPEE). October 31th -November 01st, Porto, 2013.

[3] Alves, G.R., Marques, M.A., Viegas, C., Costa Lobo, M.C. "Using VISIR in a large undergraduate course: Preliminary assessment results", Proceedings of Global Engineering Education Conference (EDUCON), 2011.

[4] Becker, J, Trendelenburg, S., Henrici,F., Manoli, Y. , "A field programmable Gm-C filter array (FPAA), Proceedings of the Second NASA/ESA Conference on Adaptive Hardware and Systems(AHS), 2007.

[5] Anadigm, http://www.anadigm.com/, Visited in January 2015.

Cypress - Programmable System-on-Chip, http://www.cypress.com/psoc/? source=CY-ENG-HEADER, Visited in January 2015. 